\title{
The prognostic time dependence of intra-tumoural IFN $\gamma$ mRNA and protein in patients with breast cancer followed for 14 years after surgery and radiotherapy, without subsequent systemic therapy
}

\author{
Nataša Todorović-Raković, Marko Radulovic, Tijana Vujasinović, Jelena Milovanović, \\ Dragica Nikolić-Vukosavljević \\ Department of Experimental Oncology, Institute of Oncology and Radiology of Serbia, Belgrade, Serbia \\ Correspondence: M Radulovic \\ $<$ marko@radulovic.net $>$ \\ Accepted for publication December 21, 2017
}

To cite this article: Todorović-Raković N, Radulovic M, Vujasinović T, Milovanović J, Nikolić-Vukosavljević D. The prognostic time dependence of intra-tumoural IFN mRNA and protein in patients with breast cancer followed for 14 years after surgery and radiotherapy, without subsequent systemic therapy. Eur. Cytokine Netw. 2017; 28(4): 151-6 doi:10.1684/ecn.2018.0402

\begin{abstract}
There is increasing evidence for the importance of immunity in breast cancer. IFN $\gamma$ is expected to have a prognostic value based on its major role in innate and specific cell-mediated immunity. In this retrospective study, based on the 14-year follow-up of 73 patients with breast cancer after surgery and radiotherapy but no subsequent systemic therapy, we investigated the prognostic time dependence of intra-tumoural IFN $\gamma$ mRNA and protein levels. Over the entire 14 years of follow-up, neither IFN $\gamma$ mRNA nor protein was significantly associated with metastasis outcome by AUC and Cox regression criteria. However, evaluation of the shorter periods has revealed a prognostic significance in the late follow-up period of 7-14 years for IFN $\gamma$ mRNA and protein with the maximal respective AUCs of 0.72 and 0.73 and hazard ratios of 6.1 and 5.2, respectively. Interestingly, the opposite prognostic association was discovered for IFN $\gamma$ mRNA and protein in the first 7 years of follow-up, possibly due to the negative correlation of IFN $\gamma$ protein and mRNA. Moreover, the prognostic association of IFN $\gamma$ mRNA has shifted from marking the favourable outcome in the first 7 years to poor outcome thereafter. This study contributes to clarification of the previously inconsistent prognostic performance of IFN $\gamma$ by providing the first prognostic evaluation with long follow-up, time-dependence assessment and absence of any chemotherapy influence.
\end{abstract}

Key words: prognosis, metastasis, breast cancer, cytokine, inflammation, interferon gamma

Interferon gamma (IFN $\gamma$ ) is expected to have a prognostic value in cancer based on its major role in innate and specific cell-mediated immunity [1], transcriptional control [2], cancer immunoediting [3, 4] and tumour immune surveillance. The action of IFN $\gamma$ in anti-tumour immunity is complex and contradictory, as both tumour-promoting and tumour-suppressing activities have been attributed to IFN $\gamma$. The exact molecular mechanism responsible for the pleiotropic IFN- $\gamma$ function in anti-tumour immunity is not yet clear [5]. Anti-tumour effects include evidence that IFN $\gamma$ immunotherapy leads to tumour regression in humans [6] and mice [7], while higher IFN $\gamma$ levels in serum prognosticates a favourable breast cancer outcome [8]. On the other hand, evidence regarding the pro-tumourigenic

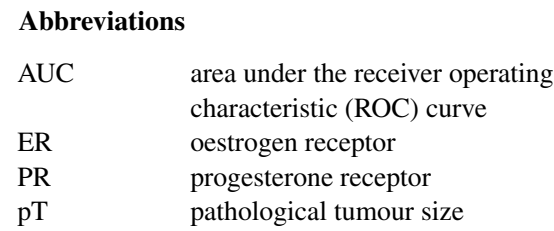

role of IFN $\gamma$ includes its promotion of lung cancer progression [5].

The objective of this retrospective study was to clarify the contradictory prognostic performance of intra-tumoural IFN $\gamma$ in breast cancer by the first investigation at both mRNA and protein levels. The time dependence of prognostic association could also be evaluated due to a very long follow-up. Furthermore, the absence of systemic treatments that could interfere with metastasis occurrence is the optimal way to characterize the prognostic value of a potential biomarker.

\section{METHODS}

\section{Patients}

We retrospectively evaluated 73 patients with breast cancer who underwent a primary tumour removal surgery at the Institute for Oncology and Radiology in the year 1993. The study was approved by the Institutional Review Board and conforms with the Code of Ethics of the World 
Medical Association (Declaration of Helsinki) printed in the British Medical Journal (18 July 1964) and its 7th revision in 2013. Patient data were received in a de-identified and recoded form without direct or indirect identifiers that could enable re-identification. This report was written according to REMARK recommendations for tumour marker prognostic studies [9]. This group of patients with invasive breast cancer was node negative and without any hormonal or chemotherapeutic systemic treatment that could interfere with metastasis occurrence. We assembled this very specific patient group from a period of over 20 years ago when patients with low risk for metastasis were not prescribed systemic therapy at our institution. This was in line with recommendations valid in the year 1993 for the pT1/2 and N0M0 patients.

The prospective power calculation rested on a pilot experiment that included 26 patients. The area under the receiver operating characteristic (ROC) curve (AUC)based prospective power calculation for the AUC of 0.75 achieved in the pilot experiment and $25 \%$ event rate calculated a requirement for a total of 39 patients with 7 events (MedCalc Software, Ostend, Belgium). The final sample size amounted to 73 patients with 21 events, with an actual event rate of $29 \%$.

The median follow-up period for patients without metastasis occurrence was 167 months, while the median time for distant metastasis occurrence from the date of primary tumour removal surgery was 38 months. The average age at diagnosis was 58 years. Fifty-three percent of patients were $\mathrm{pT} 1$, and $47 \%$ were pT2+3. Among the patients, $90 \%$ belonged to grade II. Seventy-nine percent of patients were postmenopausal (peri + post), and $21 \%$ were premenopausal. Oestrogen and progesterone receptors were determined by a dextran-coated charcoal method as described. Twenty-six percent of patients were ERnegative, while $75 \%$ were PR-negative.

\section{Real-Time PCR Assay}

Samples of breast tumour tissue with an approximate volume of $2 \mathrm{~mm}^{3}$ were homogenized on ice in the presence of ceramic microbeads for 60 seconds with an MP Fast Prep 24 homogenizer in 600- $\mu$ l guanidinium thiocyanate solution (RLT buffer, Qiagen Inc., Santa Clarita, $C A$ ) supplemented with $0.1 \mathrm{M} 2$-mercaptoethanol. The homogenate was further processed by centrifugation for 2 min at $12.000 \times \mathrm{g}$ in a QIA shredder homogenizer (Qiagen).

Total RNA was then isolated with the RNeasy mini kit (Qiagen). The integrity of the obtained RNA was examined by use of the Agilent RNA 6000 Nano kit (Agilent Technologies, Santa Clara, CA). Only RNA with the integrity number greater than 5 was subsequently reverse transcribed with the High-Capacity cDNA reverse transcription kit (Thermo Fisher Scientific, Waltham, MA) by use of hexanucleotide random primers.

Quantitative PCR was performed with the TaqMan Universal PCR Master Mix, No AmpErase UNG kit containing AmpliTaq Gold DNA polymerase (Thermo Fisher Scientific). The Hs00989291_m1 IFN $\gamma$ TaqMan probe was used for amplification. This probe spans exons. Transcripts were amplified for 40 cycles for $15 \mathrm{~s}$ at $95^{\circ} \mathrm{C}$ and for $60 \mathrm{~s}$ at $60^{\circ} \mathrm{C}$ by a $7900^{\mathrm{TH}}$ TaqMan robot (Thermo Fisher Scientific). 18S rRNA was used as a normalization control for
mRNA input. Only samples with a $\mathrm{Ct}<15$ for $18 \mathrm{~S}$ rRNA were considered for further analysis.

\section{ELISA assay}

The amount of IFN $\gamma$ protein was determined in breast tumour cytosols by Human IFN-gamma Quantikine ELISA Kit, R\&D Systems, Minneapolis, MN. Results are expressed in picograms per milligram of cytosol protein. The total amount of proteins in cytosol was determined by the Lowry method [10].

\section{Data categorization}

The outcome-oriented categorization of continuous values was achieved by use of the optimal cut-off points identified with the maximal chi-square method, X-tile 3.6.1 software from Yale University, New Haven, CT [11]. Pathological tumour size (pT) was categorized by the clinically established criteria for this parameter $(<2 \mathrm{~cm}, \mathrm{pT}=1$; $2-5 \mathrm{~cm}, \mathrm{pT}=2$ ). On the basis of the IFN $\gamma$ mRNA and protein numeric values and aforementioned cut-off points, the patients were divided into high- and low-risk groups.

\section{Evaluation of prognostic performance and time dependence}

AUC values were calculated as a quantitative measure of prognostic discrimination efficiency. Univariate Cox proportional hazards regression test was employed as an additional measure of the association between the prognosticated and actual metastasis outcomes. The hazard ratio (HR) designates the effect size of the Cox proportional hazards regression, corresponding to metastasis rates in highand low-risk groups of patients (calculated by SPSS version 23 software, IBM SPSS Statistics, Chicago, IL). The proportional hazards assumption was tested for each feature by the Cox proportional hazards test for time-dependent covariates. The assumption is satisfied if the interaction of the feature $(\mathrm{F})$ with its product with time $\left(\mathrm{F}^{*} \mathrm{~T}\right)$ reveals $\mathrm{p}>0.05$ for $\mathrm{F}^{*} \mathrm{~T}$. Proportionality assumption was further evaluated by Schoenfeld residuals and graphical evaluation as the second opinion tests, estat phtest and stphplot, respectively, in Stata/MP 13 (StataCorp, College Station, TX).

\section{Validation strategies}

The bootstrap with 1000 random data re-samples was applied to quantify the optimism bias (Stata/MP 13 software for AUC; SPSS v23 software for Cox regression). This procedure tests model stability and reliability by estimating the bias and then corrects the bias by modification of the original AUC confidence intervals (95\% CIs) as previously explained in detail (Efron, 1979). The bias is the difference between the calculated uncorrected $95 \%$ CI for AUC and true $95 \%$ CI values. The advantage of bootstrap over the split-sample cross-validation as another major internal validation method is that the entire dataset is used for model development.

\section{RESULTS}

Intra-tumoural IFN $\gamma$ mRNA and protein levels were evaluated for association with distant metastasis occurrence 
Table 1

The prognostic performance of IFN $\gamma$ mRNA and protein

\begin{tabular}{|c|c|c|c|c|c|c|}
\hline Parameter & $\begin{array}{l}P \text {-value; } A U C \\
95 \% C I^{a}\end{array}$ & $\begin{array}{l}P \text {-value; Cox HR } \\
95 \% \mathrm{CI}^{\mathrm{b}}\end{array}$ & $\begin{array}{l}P \text {-value; } A U C \\
95 \% C I^{a}\end{array}$ & $\begin{array}{l}P \text {-value; Cox HR } \\
95 \% \mathbf{C I}^{\mathrm{b}}\end{array}$ & $\begin{array}{l}P \text {-value; } A U C \\
95 \% C I^{a}\end{array}$ & $\begin{array}{l}P \text {-value; Cox HR } \\
95 \% \text { CI }^{b}\end{array}$ \\
\hline \multirow{3}{*}{ Age } & \multicolumn{2}{|c|}{$0-14$ years; $n=72$} & \multicolumn{2}{|c|}{$0-7$ years; $n=65$} & \multicolumn{2}{|c|}{$7-14$ years; $n=62$} \\
\hline & $0.31 ; 0.58$ & $0.001 * ; 26.9$ & $0.51 ; 0.58$ & $0.20 ; 0.46$ & $0.58 ; 0.57$ & $0.03^{*} ; 5.9$ \\
\hline & $0.43-0.72$ & $20.1-36.2$ & $0.40-0.75$ & $0.07-2.5$ & $0.36-0.80$ & $1.3-99.5$ \\
\hline \multirow[t]{2}{*}{$\mathrm{pT}$} & $0.13 ; 0.62$ & $0.09 ; 2.9$ & $0.14 ; 0.64$ & $044 ; 1.7$ & $0.18 ; 0.69$ & $0.12 ; 2.9$ \\
\hline & $0.49-0.79$ & $1.1-21.1$ & $0.47-0.82$ & $0.44-44.7$ & $0.40-0.87$ & $1.9-121.5$ \\
\hline \multirow[t]{2}{*}{ ER } & $0.12 ; 0.63$ & $0.02 * ; 2.7$ & $0.14 ; 0.67$ & $0.05^{*} ; 2.9$ & $0.63 ; 0.58$ & $0.22 ; 2.4$ \\
\hline & $0.47-0.79$ & $0.99-7.1$ & $0.48-0.86$ & $0.74-21$ & $0.30-0.82$ & $0.04-12.2$ \\
\hline \multirow{2}{*}{$\begin{array}{l}\mathrm{IFN} \gamma \\
\text { protein }\end{array}$} & $0.06 ; 0.59$ & $0.06 ; 3.1$ & $0.07 ; 0.60$ & $0.09 ; 4.5$ & $0.01^{* *} ; 0.73$ & $0.01^{*} ; 5.2$ \\
\hline & $0.46-0.70$ & $1.1-36.6$ & $0.46-0.73$ & $0.95-544$ & $0.58-0.85$ & $0.95-33.1$ \\
\hline $\mathrm{IFN} \gamma$ & $0.26 ; 0.40$ & $0.13 ; 0.50$ & $0.03 * * ; 0.29$ & $0.01 * ; 0.17$ & $0.09 ; 0.72$ & $0.05^{*} ; 6.1$ \\
\hline mRNA & $0.25-0.57$ & $0.03-1.2$ & $0.13-0.46$ & $0.01-1.1$ & $0.51-0.92$ & $1.7-121.5$ \\
\hline
\end{tabular}

a ROC analysis, bootstrap corrected

${ }^{\mathrm{b}}$ Cox proportional hazards regression test, bootstrap corrected

* significant by Cox proportional hazards regression analysis; $P<0.05$

** significant by the ROC analysis; $P<0.05$

to examine their prognostic value in breast cancer. In this study, we use the term 'metastasis-free survival' to indicate our measurement of time from tumour-removal surgery until the occurrence of distant metastasis. The more usual 'progression-free survival' term does not discriminate between the local tumour re-growth and distant metastasis as progression events. We chose distant metastasis occurrence as the endpoint of this study because metastasis is the predominant cause of death and thus the defining event of this disease. Another usual 'overall survival' endpoint would have included a systemic cytotoxic therapy prescribed to treat metastasis, thus eliminating the major advantage of the current study, which is the absence of systemic treatments.

Table 1 presents a statistical prognostic evaluation of several standard demographic and clinicopathological features, together with IFN $\gamma$ mRNA and protein levels for the early (0-7 years), late (7-14 years), and the entire follow-up of 0-14 years. These three different periods are shaded in Table 1 for easier orientation. The prognostic significance was evaluated by use of Cox proportional hazards regression and AUC analysis, which are presented in adjacent columns for each of the three follow-up periods (table 1). All tests were corrected for bias by the bootstrap internal validation (table 1).

The direction of association with metastasis outcome in Table 1 is indicated both by HR (null hypothesis = 1) and AUC values (null hypothesis $=0.5$; Table 1 ). Thereby, $\mathrm{HR}<1$ indicates good prognosis, and $\mathrm{HR}>1$ indicates poor prognosis, while $\mathrm{AUC}<0.5$ designates good prognosis, and $\mathrm{AUC}>0.5$ indicates poor prognosis.

The prognostic significance of each statistical test and follow-up period is indicated by a $P$-value (table 1 ). IFN $\gamma$ mRNA and protein did not show a significant prognostic association over the entire 0-14-year follow-up (table 1). However, a division of the entire follow-up has revealed significant prognostic associations for the shorter periods. More precisely, IFN $\gamma$ protein was significantly associated with poor outcome in the late 7-14-year follow-up $(P=0.01)$, while IFN $\gamma$ mRNA was associated significantly with good outcome in early follow-up $(P=0.01-0.03)$ and then switched to a significant association with poor out- come during late follow-up $(P=0.05-0.09$; table 1 and figures 1,2).

In addition to $P$-values, prognostic performance was additionally estimated by HR and AUC values. HR of 0.17 was the furthest removed from its null hypothesis value of 1.0 (table 1). It was achieved by IFN $\gamma$ mRNA during 0-7 years, followed by IFN $\gamma$ mRNA with HR of 6.1 and IFN $\gamma$ protein with HR of 5.2, during 7-14 years (table 1). The most pronounced AUCs were achieved by IFN $\gamma$ mRNA (0.72) and protein $(0.73)$ in the period of 7-14 years (table 1 ).

Kaplan-Meier estimator plots illustrate an association of IFN $\gamma$ mRNA and protein with metastasis outcome for different follow-up periods (figure 1). Upper and lower curves represent the two patient groups defined by an optimal cutoff point. A wider separation between the upper and lower curves indicates a better prognostic performance. Patients with higher risk are designated on the lower line, as their probability of remaining metastasis free is lower (figure 1). Dotted lines indicate the patient group with higher IFNy values. Taken together, dotted line below a full line indicates an association with higher risk (figure $1 A, B, C, F$ ), while the dotted line positioned above indicates an association with lower risk (figure 1D,E). Figure 1 illustrates an apparent but not significant prognostic divergence of IFN $\gamma$ mRNA and protein levels for the early 0-7-year (figure $1 B, E$ ) and the entire 0-14-year (figure 1A,D) periods. Such divergence disappeared in the late follow-up period (figure $1 C, F$ ). Figure 1 also illustrates the statistically significant prognostic shift of IFN $\gamma$ mRNA levels (figure $1 E, F)$. However, the time-dependent prognostic value could not be confirmed for IFN $\gamma$ mRNA by Cox regression with time-dependent covariates $(p=0.16$ for T_cov_) and Schoenfeld residuals $(p=0.81)$.

Figure 2 illustrates the distribution of individual measured values for IFN $\gamma$ mRNA and protein. It is obvious that metastatic patients have on average higher IFN $\gamma$ protein values (figure $2 A$ ), which is in agreement with results presented in Table 1 and Figure 1, indicating that IFN $\gamma$ protein was a marker of higher disease risk. Data presented in Figure $2 B$ show lower IFN $\gamma$ mRNA values in patients with early metastasis and higher values in those with late 


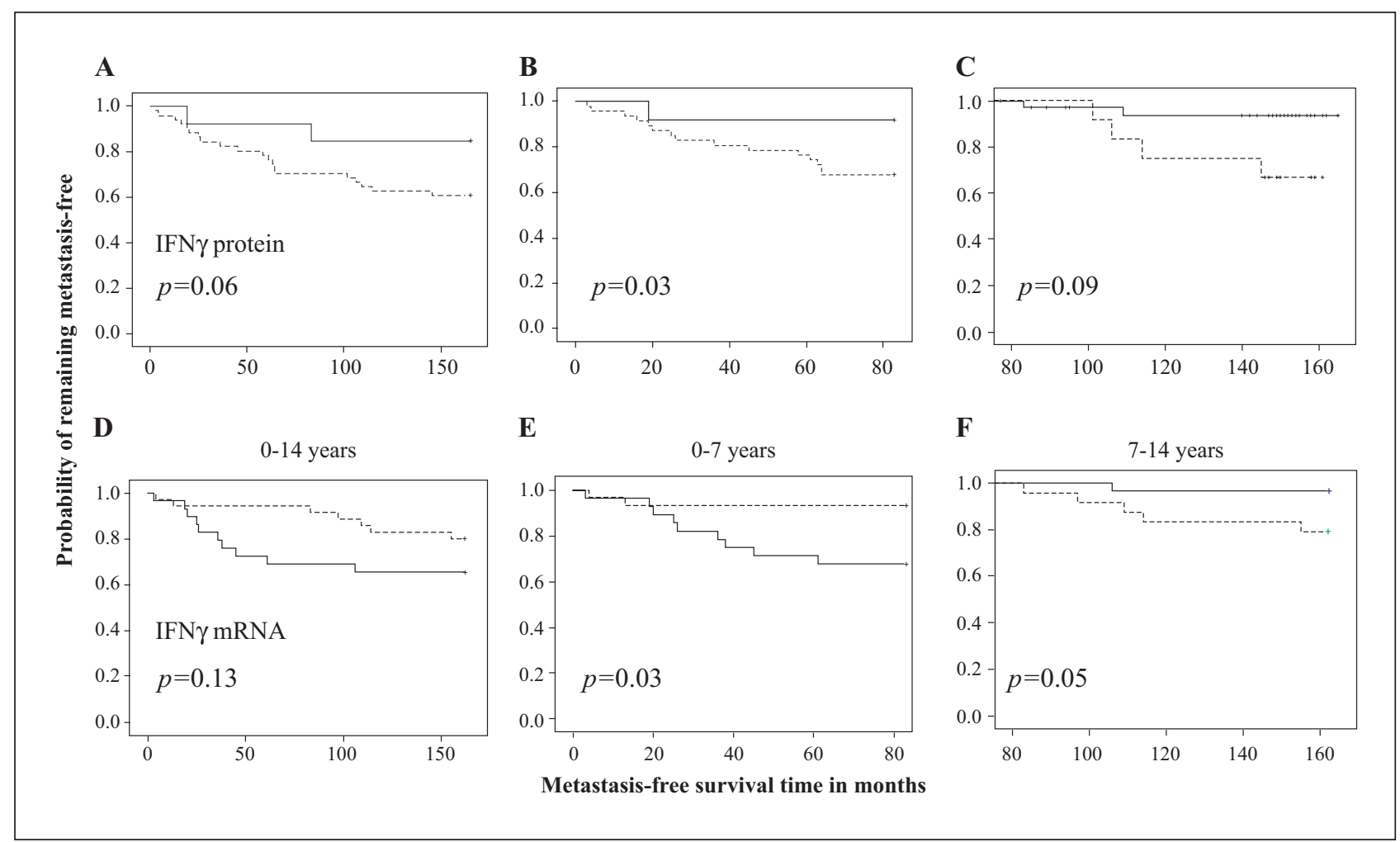

Figure 1

Kaplan-Meier analysis of the IFN $\gamma$ prognostic performance. Plots represent A) IFN $\gamma$ protein during the entire 0-14-year follow-up period. B) IFN $\gamma$ protein during the first 7 years of follow-up. C) IFN $\gamma$ protein during the late 7-14 years of follow-up. D) IFN $\gamma$ mRNA during 0-14 years. E) IFN $\gamma$ mRNA during 0-7 years. F) IFN $\gamma$ mRNA during 7- 14 years. High-feature values are plotted on dotted lines, while lower values are presented by solid lines. Plots were drawn by values categorized with an optimal threshold. $P$-values were calculated by the Cox proportional hazards regression test.

Table 2

Correlations between IFN $\gamma$ mRNA, IFN $\gamma$ protein, oestrogen and progesterone receptor levels ${ }^{\text {a }}$

\begin{tabular}{|llll|}
\hline \multicolumn{5}{l}{ IFN $\gamma$ protein } & ER & PR \\
\hline \multicolumn{5}{c}{$0-14$ years } \\
\hline IFN $\gamma$ mRNA & $-0.43^{*}$ & -0.25 & $-0.35^{*}$ \\
\hline IFN $\gamma$ protein & - & -0.05 & -0.08 \\
\hline \multicolumn{5}{c}{$0-7$ years } \\
\hline IFN $\gamma$ mRNA & $-0.40^{*}$ & -0.18 & -0.31 \\
\hline IFN $\gamma$ protein & - & -0.01 & -0.09 \\
\hline & & $7-14$ years \\
\hline IFN $\gamma$ mRNA & $-0.59^{*}$ & $-0.31^{*}$ & $-0.38^{*}$ \\
\hline IFN $\gamma$ protein & - & -0.01 & -0.04 \\
\hline
\end{tabular}

${ }^{a}$ Spearman's rank order correlation coefficients are indicated ${ }^{*} p<0.05$

metastasis, which is again in line with data in Table 1 and Figure 1.

A significant negative correlation was noted between IFN $\gamma$ protein and mRNA expression levels with a Spearman's coefficient of -0.43 over the entire follow-up (table 2). Furthermore, IFN $\gamma$ mRNA showed a significant negative correlation with PR (Spearman's coefficient $=-0.35$ ), while IFN $\gamma$ protein did not correlate significantly with steroid receptor levels (table 2). Similar correlation profiles were also noted for the 0-7- and 7-14-year periods (table 2).

\section{DISCUSSION}

This study examined the prognostic value of intra-tumoural IFN $\gamma$ mRNA and protein levels in breast cancer. While intra-tumoural IFN $\gamma$ protein exerted a consistent prognostic association with poor disease outcome, our results for the first time reveal a peculiar time-dependent prognostic shift for the IFN $\gamma$ mRNA.

An association between IFN $\gamma$ protein and mRNA with metastasis occurrence was weak when analysed throughout the 14-year follow-up. Having in mind that IFN $\gamma$ is functionally pleiotropic [12] and that prognostic performance can be time dependent [13], we took advantage of the long follow-up and performed additional prognostic analysis in the two shorter periods. Such analysis has revealed a much stronger prognostic association for both IFN $\gamma$ protein and mRNA in 0-7- and 7-14-year time frames.

The finding that IFN $\gamma$ mRNA was a marker of good outcome in the period of 0-7 years was expected, as this cytokine is considered to have a pronounced anti-cancer activity based on results showing that decreased IFN $\gamma$ levels or its genetic defects are risk factors for tumourigenesis in humans [14]. Marth et al. similarly showed that elevated levels of IFN $\gamma$ were associated with improved clinical outcome in patients with ovarian cancer [15]. Moreover, IFN $\gamma$ also exerts direct anti-tumour effects, including anti-angiogenic, anti-proliferative, pro-apoptotic and immunostimulatory [16]. Previous prognostic studies have shown that IFNy mRNA correlates with favourable 


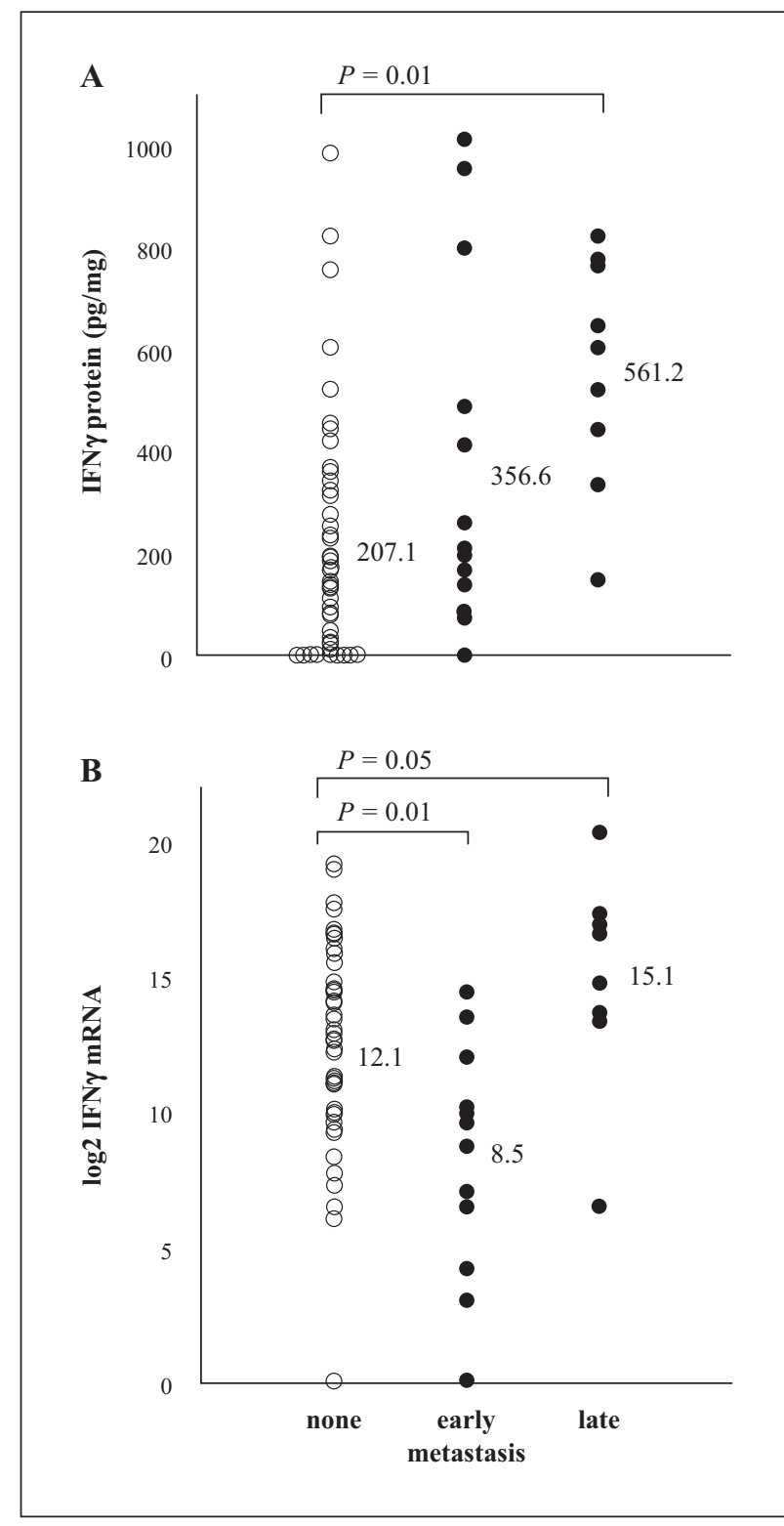

Figure 2

Comparison of IFN $\gamma(\mathbf{A})$ mRNA and (B) protein levels in patients without metastasis, early (0-7 years) metastasis and late (7-14 years) metastasis. Average values are indicated for each column. Dots indicate individual patient values in $\mathrm{pg} / \mathrm{mg}$ for IFN $\gamma$ protein or a $\log 2$ scale for mRNA levels expressed in arbitrary units relative to expression of 18S rRNA, which was used as a normalization control for mRNA input. $P$-values were calculated by Cox proportional hazards regression analysis.

clinical outcome in ovarian cancer [15] and cervical carcinoma [17]. These reports were consistent with our result for IFN $\gamma$ mRNA in the first 7 years of follow-up but not with the later unexpected and previously unreported shift to association with poor outcome. The time-shifting prognostic effects in breast cancer have been previously demonstrated for the hormone receptor levels, tumour size and histologic grade [13], but have not been reported for IFN $\gamma$. The late association of IFN $\gamma$ mRNA with poor outcome may not have been observed in the aforementioned previous studies, which reported an association with favourable outcome due to their shorter follow-up. However, the association of IFN $\gamma$ with poor outcome was not surprising in view of its known pleiotropic function, manifested by the opposing actions depending on the cellular, microenvironmental or molecular context [3]. The peculiar prognostic swing observed for IFN $\gamma$ mRNA in this study might not indicate a change of the intra-tumoural IFNy function during the course of the disease due to the fact that IFN $\gamma$ protein did not produce the same shifting performance, probably due to post-translational effects. This possibility is partially supported by the negative correlation of mRNA and protein levels throughout the follow-up. Such phenomenon of discordant gene and protein expression was surprising, as it is generally expected that mRNA and protein levels positively correlate. However, the largescale gene expression study has provided evidence that only $40 \%$ of changes in protein levels can be explained by mRNA levels [18]. The cellular abundance of proteins is thus predominantly controlled at the level of translation. In the case of IFN $\gamma$, it can be speculated that this control might be based on conserved UA-rich sequence found in the 3' untranslated region of cytokine mRNAs, as these 'instability sequences' tightly influence the translation process [19]. Moreover, the post-transcriptional regulation may be altered or even damaged in cancer [20] based on a lower concordance between mRNA and protein levels in cancer tissue in comparison to normal tissues [21].

Although the prognostic association of IFN $\gamma$ mRNA was time shifting, its other measured relations were steady throughout the follow-up, such as inverse correlations with PR and IFN $\gamma$ protein content. The correlation with PR was in accordance with the previous study of Chavey et al., who showed that IFN $\gamma$ inversely correlated with ER and PR expression [22].

The association of high levels of IFN $\gamma$ protein with the poor outcome observed in this study may not be simply interpreted as an indication of its tumour-promoting role, in view of the report that IFN $\gamma$ signalling defects occur in lymphocytes of patients with breast cancer [23]. Therefore, the association of elevated intra-tumoural IFN $\gamma$ protein in patients with poor outcome could indicate its pro-tumourigenic role only if supported by evidence of its increased signalling. The association of IFN $\gamma$ protein with the elevated disease risk obtained in the current study was in line with the reports that PD-L1, a protein upregulated by IFN $\gamma$, was also associated with poor survival in patients with breast cancer [24]. Taken together, the activity of IFN $\gamma$ in breast tumour might have been mediated by PD-L1, even though PD-L1 upregulation is also upregulated by other cytokines such as TNF $\alpha$ [25] and IL-1 $\beta$ [26]. Nevertheless, the prognostic value of IFN $\gamma$ in breast cancer can be fully characterized without knowledge of its functional activity and the function of its downstream receptors and signalling.

Retrospective data and a relatively small sample size of 73 patients are the main limitations of this study. Division of the time frame to 0-7 and 7-14 years has further reduced patient numbers; however, these reduced sample sizes still remained above the requirements set by the sample size analysis. Advantages of this study include the patient group without any adjuvant systemic treatments, which could mask or alter the prognostic role of cytokines. The long follow-up and analysis of time dependence are the major additional advantages of this investigation.

In conclusion, we report an intriguing prognostic association of intra-tumoural IFN $\gamma$ mRNA and protein in breast cancer. To our knowledge, this is the first analysis of IFN $\gamma$ mRNA and protein levels in primary breast tumours not 
affected by any kind of systemic therapy. Novel prognostic tools may offer clinical usefulness by identifying patients who have the highest risk of early distant metastasis and death.

Disclosure. Financial support: This work was supported by the Grant from the Ministry of Science and Technological Development of the Republic of Serbia (grant number ON175068). Conflict of interest: none.

\section{REFERENCES}

1. Annunziato F, Romagnani C, Romagnani S. The 3 major types of innate and adaptive cell-mediated effector immunity. J Allergy Clin Immunol 2015; 135: 626.

2. Gough DJ, Levy DE, Johnstone RW, Clarke CJ. IFNgamma signaling-does it mean JAK-STAT? Cytokine Growth Factor Rev 2008; 19: 383 .

3. Zaidi MR, Merlino G. The two faces of interferon-gamma in cancer. Clin Cancer Res 2011; 17: 6118.

4. Dunn GP, Koebel CM, Schreiber RD. Interferons, immunity and cancer immunoediting. Nature Rev Immunol 2006; 6: 836.

5. Zhang X, Zeng Y, Qu Q, et al. PD-L1 induced by IFN-gamma from tumour-associated macrophages via the JAK/STAT3 and PI3K/AKT signaling pathways promoted progression of lung cancer. Int J Clin Oncol 2017; 6: 1026.

6. Parker BS, Rautela J, Hertzog PJ. Antitumour actions of interferons: implications for cancer therapy. Nat Rev Cancer 2016; 16: 131.

7. Kim OY, Park HT, Dinh NTH, et al. Bacterial outer membrane vesicles suppress tumour by interferon-gamma-mediated antitumour response. Nat Commun 2017; 8: 626.

8. Zhu X, Du L, Feng J, Ling Y, Xu S. Clinicopathological and prognostic significance of serum cytokine levels in breast cancer. Clin Lab 2014; 60: 1145

9. Altman DG, McShane LM, Sauerbrei W, Taube SE. Reporting recommendations for tumour marker prognostic studies (REMARK): explanation and elaboration. PLoS Med 2012;9: e1001216.

10. Lowry OH, Rosebrough NJ, Farr AL, Randall RJ. Protein measurement with the Folin phenol reagent. J Biol Chem 1951; 193: 265.

11. Camp RL, Dolled-Filhart M, Rimm DL. X-tile: a new bio-informatics tool for biomarker assessment and outcome-based cut-point optimization. Clin Cancer Res 2004; 10: 7252

12. Varela N, Munoz-Pinedo C, Ruiz-Ruiz C, Robledo G, Pedroso M, Lopez-Rivas A. Interferon-gamma sensitizes human myeloid leukemia cells to death receptor-mediated apoptosis by a pleiotropic mechanism. J Biol Chem 2001; 276: 17779.

13. Bellera CA, MacGrogan G, Debled M, de Lara CT, Brouste V, Mathoulin-Pelissier S. Variables with time-varying effects and the Cox model: some statistical concepts illustrated with a prognostic factor study in breast cancer. BMC Med Res Method 2010; 10: 20.

14. Shankaran V, Ikeda H, Bruce AT, et al. IFNgamma and lymphocytes prevent primary tumour development and shape tumour immunogenicity. Nature 2001; 410: 1107

15. Marth C, Fiegl H, Zeimet AG, et al. Interferon-gamma expression is an independent prognostic factor in ovarian cancer. Am J Obstet Gynecol 2004; 191: 1598.

16. Razaghi A, Owens L, Heimann K. Review of the recombinant human interferon gamma as an immunotherapeutic: impacts of production platforms and glycosylation. J Biotechnol 2016; 240: 48

17. Tartour E, Gey A, Sastre-Garau X, Lombard Surin I, Mosseri V, Fridman WH. Prognostic value of intratumoural interferon gamma messenger RNA expression in invasive cervical carcinomas. J Natl Cancer Inst 1998; 90: 287.

18. Schwanhausser B, Busse D, Li N, et al. Global quantification of mammalian gene expression control. Nature 2011; 473: 337.

19. Kruys V, Marinx O, Shaw G, Deschamps J, Huez G. Translational blockade imposed by cytokine-derived UA-rich sequences. Science 1989; 245: 852 .

20. Perl K, Ushakov K, Pozniak Y, et al. Reduced changes in protein compared to mRNA levels across non-proliferating tissues. $B M C$ Genomics 2017; 18: 305.

21. Kosti I, Jain N, Aran D, Butte AJ, Sirota M. Cross-tissue analysis of gene and protein expression in normal and cancer tissues. Sci Rep 2016; 6: 24799.

22. Chavey C, Bibeau F, Gourgou-Bourgade S, et al. Oestrogen receptor negative breast cancers exhibit high cytokine content. Breast Cancer Res 2007; 9: R15.

23. Critchley-Thorne RJ, Simons DL, Yan N, et al. Impaired interferon signaling is a common immune defect in human cancer. Proc Natl Acad Sci U S A 2009; 106: 9010 .

24. Guo Y, Yu P, Liu Z, et al. Prognostic and clinicopathological value of programmed death ligand-1 in breast cancer: a meta-analysis. PLoS One 2016; 11: e0156323.

25. Wu YY, Lin CW, Cheng KS, et al. Increased programmed deathligand-1 expression in human gastric epithelial cells in Helicobacter pylori infection. Clin Exp Immunol 2010; 161: 551.

26. Youngnak-Piboonratanakit $\mathrm{P}$, Tsushima $\mathrm{F}$, Otsuki $\mathrm{N}$, et al. The expression of B7-H1 on keratinocytes in chronic inflammatory mucocutaneous disease and its regulatory role. Immunol Let 2004; 94: 215. 